\title{
Recursos utilizados por graduandos de odontologia e Cirurgiões-Dentistas do SUS para estudar e resolver dúvidas surgidas durante o trabalho
}

Sonia Gomes de Lemos ${ }^{*}$ 'Maria Celeste Morita ${ }^{* *}$ 'Elisa Emi Tanaka Carloto ${ }^{* * *}$

$\begin{array}{ll}* & \text { Mestre em Odontologia, Universidade Estadual de Londrina } \\ * * & \text { Professora Associada do Departamento de Medicina Oral e Odonto- } \\ & \text { logia Infantil da Universidade Estadual de Londrina } \\ *^{* * *} & \text { Professora Associada do Departamento de Medicina Oral e Odonto- } \\ & \text { logia Infantil da Universidade Estadual de Londrina }\end{array}$

\section{RESUMO}

O objetivo foi identificar ferramentas que um grupo de 45 estudantes e 21 CirurgiõesDentistas (CD), atuantes no Sistema Único de Saúde (SUS) e preceptores de alunos, utilizam para estudar e resolver dúvidas surgidas durante o trabalho. Buscou-se também identificar barreiras para a utilização de ambientes virtuais como estratégia de apoio. Aplicou-se um questionário semiestruturado. Os resultados foram analisados por estatística descritiva e Análise de Conteúdo. Estudantes indicaram preferencialmente periódicos eletrônicos (89\%) e CD principalmente a participação em congressos $(90 \%)$. Ambos os grupos dominam ferramentas de informática básica, e-mail e ferramentas digitais de busca. Sites e blogs são utilizados por $14.3 \%$ dos CD e $17.8 \%$ dos estudantes. Nenhum CD e apenas 1 estudante indicou utilizar sites de Odontologia Baseada em Evidências. A leitura de textos em língua inglesa, fundamental para o acesso a informação científica, é barreira para mais de $50 \%$ em ambos os grupos. $\mathrm{Na}$ abordagem qualitativa $57 \%$ dos $\mathrm{CD}$ não relataram estudar para resolver dúvidas surgidas no trabalho, que são solucionadas principalmente com conversas entre colegas e encaminhamentos. Indica-se a Teleconsultoria como estratégia promissora para este grupo. Ainda que o perfil do egresso almejado seja generalista, a intenção de especializar-se é referida por $78 \%$ dos estudantes. Embora haja o domínio tecnológico, ressalta-se a barreira referente à falta de uma cultura de busca, que poderá comprometer a adesão a programas de apoio a distância. Indica-se a necessidade de enfatizar a constante busca qualificada da informação, para estudantes e profissionais, visando à resolubilidade e qualidade da atenção ofertada no SUS.

Descritores: Educação continuada. Educação em Odontologia. Educação à Distância. Odontologia Baseada em Evidências. Telessaúde. 


\section{INTRODUÇÃO}

O trabalho do Cirurgião-dentista na Atenção Primária à Saúde no Sistema Único de Saúde (SUS), apresenta grandes desafios, pois o serviço envolve não apenas práticas pontuais e tecnicistas, mas possui uma abordagem multidisciplinar e de interação individual e coletiva. Há necessidade de se manter um aprendizado constante, motivado pelas dúvidas e problemas arrolados no dia-a-dia do serviço e capaz de transformar as práticas preestabelecidas. ${ }^{1}$

A resolução de problemas deve preferencialmente acontecer com base no conhecimento científico, na experiência clínica do profissional e nas percepções/necessidades do paciente. $^{2} \mathrm{O}$ uso da Tecnologia de Comunicação e Informação (TIC) foi identificada e percebeu-se o grande proveito que poderia obter-se ao empregá-la como ferramenta de apoio aos profissionais dos serviços, por meio da Telessaúde. $^{3}$

Para alcançar o público e o efeito que se espera com o programa, faz-se necessário conhecer as características da população a que se destina. O objetivo desta pesquisa foi identificar quais ferramentas um grupo de cirurgiões-dentistas atuantes no SUS e um grupo de graduandos em Odontologia tem lançado mão para estudar e para resolver dúvidas surgidas durante o trabalho, e identificar possíveis barreiras para a expansão e consolidação da Teleodontologia como estratégia de apoio a esses profissionais.

\section{SOCIEDADE DA INFORMAÇÃO E INFOCULTURA}

A partir do surgimento da imprensa e com a difusão dos recursos eletrônicos e de internet, o uso de TIC culminou no surgimento de uma sociedade da informação, promovendo profundas mudanças sociais, culturais, na produção do conhecimento ${ }^{4}$, bem como se indicasua capacidade de moldar pensamentos, ações e comportamentos. 5,6

Os Ambientes Virtuais de Aprendizagem (AVA) e o uso de hipermídias (combinações de recursos de som, vídeo, hipertextos) surgem nesse cenário apontando novas possibilidades para a abordagem pedagógica e consolidação do processo ensino-aprendizagem, devido à diversidade de possibilidades de inovação e interação. ${ }^{7,8}$

Além disso, o uso das TIC é uma importante ferramenta de educação que permite quebrar barreiras geográficas (muito relevante considerando a extensão territorial brasileira), econômicas/estruturais (cada vez mais se busca a inclusão digital, como o Programa Nacional de Banda Larga - Brasil Conectado), ${ }^{9}$ barreiras espaciais, de tempo e de interatividade. ${ }^{10}$

A TIC oferece vantagens tanto como ferramenta regular de ensino em cursos presenciais, como também para profissionais no período pós-formado. Com a acelerada forma com que aparecem novas tecnologias e novos conhecimentos, exige-se do profissional constante atualização. Indica-se a necessidade do sistema educacional contemplar a "formação ao longo da vida" com sinergia ao ambiente de trabalho. ${ }^{11}$

\section{EDUCAÇÃO PERMANENTE EM SAÚDE E AS DIRETRIZES CUR- RICULARES NACIONAIS}

O enfoque na educação permanente surge nas Diretrizes Curriculares Nacionais (DCN) para o curso de graduação em Odontologia no ano de 2002, ${ }^{12}$ como uma das competências gerais para a formação profissional.

A proposta curricular sugere a quebra do modelo de ensino baseado na pers- 
pectiva bancária de acúmulo e transmissão do saber ${ }^{13}$ e do modelo flexneriano ${ }^{14}$, e implantação de um ensino voltado para a autoaprendizagem, aprender a aprender e busca autônoma do conhecimento. Vê-se um processo baseado no desenvolvimento de competências, ou seja, na capacidade do profissional/estudante mobilizar conhecimentos em diferentes áreas do conhecimento, para a resolução dos problemas concretos encontrados nas atividades diárias. ${ }^{15}$

A educação continuada do profissional é motivo de atenção pelos órgãos gestores do SUS. Com base na observação de alguns projetos brasileiros em Telessaúde e Telemedicina, o Ministério da Saúde, a partir de 2006, desenvolveu e implantou um projeto-piloto de Telessaúde. ${ }^{3}$

As ações estão voltadas para a atenção básica (em equipes da Estratégia de Saúde da Família) ${ }^{16}$ devido ao modelo de atenção, com porta de entrada na Atenção Primária à Saúde (APS). Quando bem estruturado este nível de atenção pode resolver aproximadamente $85 \%$ das necessidades em saúde da população e aliviar os níveis secundário e terciário, que demandam tecnologias de alto custo. ${ }^{17,18}$

Os objetivos são: a otimização dos recursos, redução de custos de deslocamento e tempo referente às ausências no trabalho, tornar $o$ atendimento mais rápido, melhorar a resolubilidade e qualidade do sistema e favorecer a permanência de profissionais em locais de difícil acesso.

Oferta-se apoio processual e científico aos profissionais. Os serviços prestados pelos Núcleos Técnico-científicos, representado pelas instituições formadoras e de gestão, são: ${ }^{19}$

Teleconsultorias: utiliza-se a forma síncrona (chat, web ou videoconferência) ou assíncrona (mensagens off-line) para estabelecer contato entre o profissional e o teleconsultor, que poderá resolver suas dúvidas;

Segunda Opinião Formativa: a partir das teleconsultorias realizadas, selecionamse as perguntas de maior frequência e relevância e disponibiliza-se por meio do site da Biblioteca Virtual em Saúde (BVS), no domínio BVS-APS, o conjunto de pergunta e resposta. Nesse caso, as respostas vêm acompanhadas por referências bibliográficas.

Telediagnóstico: utiliza as TIC para oferecer apoio diagnóstico à distância.

Tele-educação: oferta de cursos, conferências e aulas utilizando ambientes virtuais.

\section{METODOLOGIA}

Esta pesquisa recebeu aprovação pelo Comitê de Ética em Pesquisa Envolvendo Seres Humanos, protocolo CAAE: 13290713.3.0000.5231, autorização da Secretaria Estadual de Saúde do Estado do Paraná e do Colegiado do Curso de Odontologia da universidade onde se aplicou a pesquisa.

Realizou-se um estudo exploratório, retrospectivo de abordagem descritiva. A população de estudo foi composta por: vinte e um cirurgiões-dentistas atuantes em unidades básicas de saúde das cidades de Londrina, Cambé e Ibiporã, no estado do Paraná. Teve como critério de inclusão ser preceptor do Programa de Educação pelo Trabalho na saúde (PET-Saúde); e quarenta e cinco estudantes do último semestre da graduação em Odontologia de uma universidade de Londrina, no ano de 2013.

$\mathrm{O}$ projeto pedagógico do curso de Odontologia pesquisado possui formação de acordo com as DCN e voltada para o SUS, desde o ano de $2005 .{ }^{20}$ O PET-Saúde é parte dessa formação e, assim os $\mathrm{CD}$ que são também preceptores de alunos, foram sele- 
cionados para participar dessa pesquisa, visto sua importância como extensão do ambiente estudantil e de formação profissional, justifica-se assim a amostra de conveniência obtida.

$\mathrm{O}$ instrumento de pesquisa foi adaptado do Formulário Linhas de Base, utilizado pelo programa Telessaúde Brasil Odontologia. $^{21}$ Realizou-se um pré-teste com um representante de cada grupo, no qual se pode avaliar o tempo de resposta e a compreensão das perguntas.

Verificou-se a necessidade de melhorar a clareza e objetividade de algumas questões. Os respondentes relataram que o questionário estava muito extenso e cansativo, assim algumas questões foram removidas e/ou reformuladas.

Trata-se de um questionário semiestruturado autoaplicável. Os questionários foram entregues para os CD em seu local de trabalho e estes puderam responder em sua residência.

A aplicação do instrumento aos estudantes ocorreu durante o último semestre do curso, no segundo mês após o retorno das férias, em sala de aula no período da manhã.
Os dados foram transcritos para uma planilha em Microsoft Excel e as medidas de frequência analisadas utilizando-se o programa Epiinfo 3.5.2. Realizou-se um exame qualitativo da questão aberta, por meio da Análise de Conteúdo. ${ }^{22}$

\section{RESULTADOS}

As informações referentes ao perfil dos estudantes de graduação em Odontologia e dos CD pesquisados estão nas Tabela 1 e 2 .

Com relação a habilidades de leitura em língua estrangeira, argumentou-se: "Quão confortável você se sente para ler textos em inglês?" Havia uma escala visual, onde o respondente deveria marcar o rosto correspondente a sua resposta. Entre os CD $57.1 \%$ marcaram opções entre "Pouco confortável" e "Incompatível" e 42.9\% "Muito confortável" e "Confortável". Entre os estudantes os resultados foram semelhantes, 53.4\% marcaram entre "Pouco confortável" e "Incompatível" e 46.6\% "Muito confortável" e "Confortável". 
Recursos utilizados por graduandos de odontologia e Cirurgiões-Dentistas do SUS para estudar e resolver dúvidas surgidas durante o trabalho

Tabela 1 - Perfil dos Cirurgiões-dentistas pesquisados

\begin{tabular}{lccc}
\hline Itens analisados & Resultados & \\
\hline \multirow{2}{*}{ Sexo } & Feminino & $\mathrm{N}$ & $\%$ \\
\multirow{2}{*}{ Faixa etária } & Masculino & 15 & $71.4 \%$ \\
& & 6 & $28.6 \%$ \\
& 30 a 39 anos & 2 & $9.5 \%$ \\
Tempo de formado & 40 a 49 anos & 16 & $76.2 \%$ \\
& 50 a 59 anos & 3 & $14.3 \%$ \\
& & & \\
Concluiu curso de Atualização? & 5 a 19 anos & 4 & $19 \%$ \\
& 20 a 29 anos & 16 & $76.2 \%$ \\
& 30 a 39 anos & 1 & $4.8 \%$ \\
& Sim & 9 & $42.9 \%$ \\
& Não & 11 & $52.4 \%$
\end{tabular}

Cursos de Atualização que concluíram:

Concluiu curso de Especialização?

$\begin{array}{ccc}\text { Dentística / Estética dental } & 4 & 19 \% \\ \text { Programa Saúde da Família } & 3 & 14.3 \% \\ \text { Oclusão e disfunção da ATM } & 2 & 9.5 \% \\ \text { Ortodontia } & 2 & 9.5 \% \\ \text { Periodontia } & 2 & 9.5 \% \\ \text { Prótese } & 1 & 4.8 \% \\ \text { Saúde do Adolescente } & 1 & 4.8 \% \\ \text { Atenção a gestante } & 1 & 4.8 \% \\ \text { Ortopedia funcional } & 1 & 4.8 \% \\ & & \\ \text { Sim } & 18 * & 85.7 \% \\ \text { Não } & 2 & 9.5 \% \\ \text { NR } & 1 & 4.8 \%\end{array}$

Cursos de Especialização que concluíram:

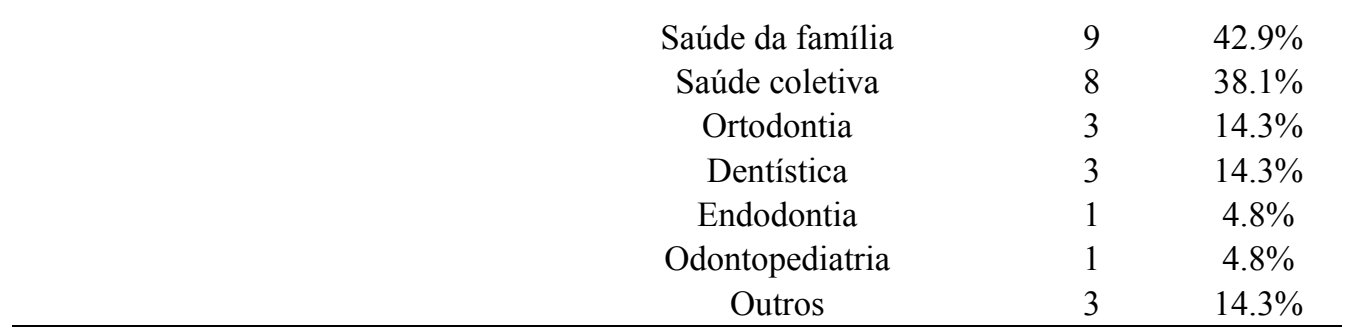

*Destes, 9 (45\%) possuem 2 especialidades

Pesquisou-se também as ferramentas que estes utilizam para estudar e se atualizar (Figura 1). Demarcou-se também os meios virtuais utilizados por cada CD respondente. Assim, observou-se que do total de $21 \mathrm{CD}$ pesquisados 17 (81\%) utili- zam pelo menos um meio virtual para estudar/atualizar. Examinou-se também a utilização de fontes de informação cientifica (periódicos, livros, etc.) e notou-se que $3(14 \%)$ respondentes não utilizam essas fontes, recorrendo apenas às discussão com colegas, grupos de estudo ou palestras. 
Recursos utilizados por graduandos de odontologia e Cirurgiões-Dentistas do SUS para estudar e resolver dúvidas surgidas durante o trabalho

Tabela 2 - Perfil dos estudantes / graduandos em Odontologia

\begin{tabular}{ccc}
\hline Resultados & \\
\hline Itens analisados & $\mathrm{N}$ & $\%$
\end{tabular}

Sexo

$\begin{array}{lcc}\text { Feminino } & 29 & 64,4 \% \\ \text { Masculino } & 15 & 33.3 \% \\ \text { NR } & 1 & 2.3 \%\end{array}$

Foi bolsista do programa PET-Saúde?

$\begin{array}{lcc}\text { Sim } & 13 & 28.9 \% \\ \text { Não } & 31 & 68.9 \% \\ \text { NR } & 1 & 2.3 \%\end{array}$

Pretende fazer curso de atualização?

$\begin{array}{lcc}\text { Sim } & 37 & 82.2 \% \\ \text { Não } & 6 & 13.3 \% \\ \text { NR } & 2 & 4.4 \%\end{array}$

Em qual área?

$\begin{array}{lcc}\text { Endodontia } & 18 & 40 \% \\ \text { Prótese } & 11 & 24.4 \% \\ \text { Periodontia } & 9 & 20 \% \\ \text { Dentística } & 6 & 13.3 \%\end{array}$

Outros (cirurgia,

farmacologia, estéti- $13 \quad 28.9 \%$

ca)

Pretende fazer curso de Especialização ou

Residência?

$\begin{array}{lll}\text { Sim } & 35 & 77.7 \% \\ \text { Não } & 10 & 22.3 \%\end{array}$

Cursos de Especialização pretendidos

\begin{tabular}{lcc} 
Ortodontia & 13 & $28.9 \%$ \\
Implantodontia & 9 & $20 \%$ \\
Endodontia & 9 & $20 \%$ \\
Prótese & 9 & $20 \%$ \\
Periodontia & 8 & $17.8 \%$ \\
Dentística & 5 & $11.1 \%$ \\
CTBMF & 4 & $8.9 \%$ \\
Saúde coletiva & 1 & $2.3 \%$ \\
Odontopediatria & 1 & $2.3 \%$ \\
\multicolumn{2}{c}{ Residência na área da saúde } \\
CTBMF & 2 & $4.4 \%$ \\
Odontopediatria & 2 & $4.4 \%$ \\
\multicolumn{2}{r}{ Residência multiprofissional } \\
Saúde da Família & 1 & $2.3 \%$
\end{tabular}


Recursos utilizados por graduandos de odontologia e Cirurgiões-Dentistas do SUS para estudar e resolver dúvidas surgidas durante o trabalho

Figura 1 - Gráfico representativo das ferramentas que utilizam para estudar / atualizar

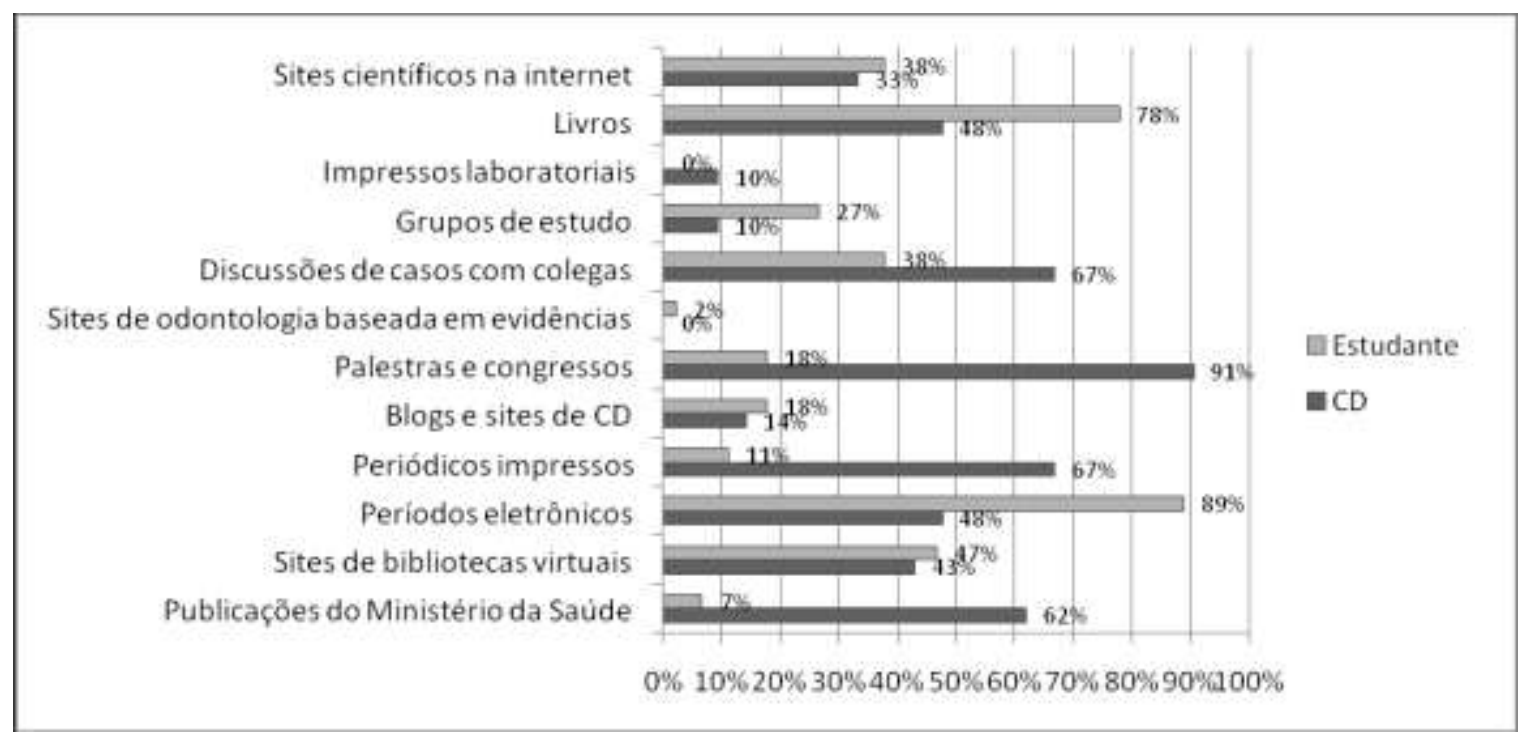

Na Figura 2 estão expostos os resultados referentes a programas e aplicativos para computador que os pesquisados sabem utilizar.

Figura 2 - Gráfico das ferramentas de informática que sabem utilizar

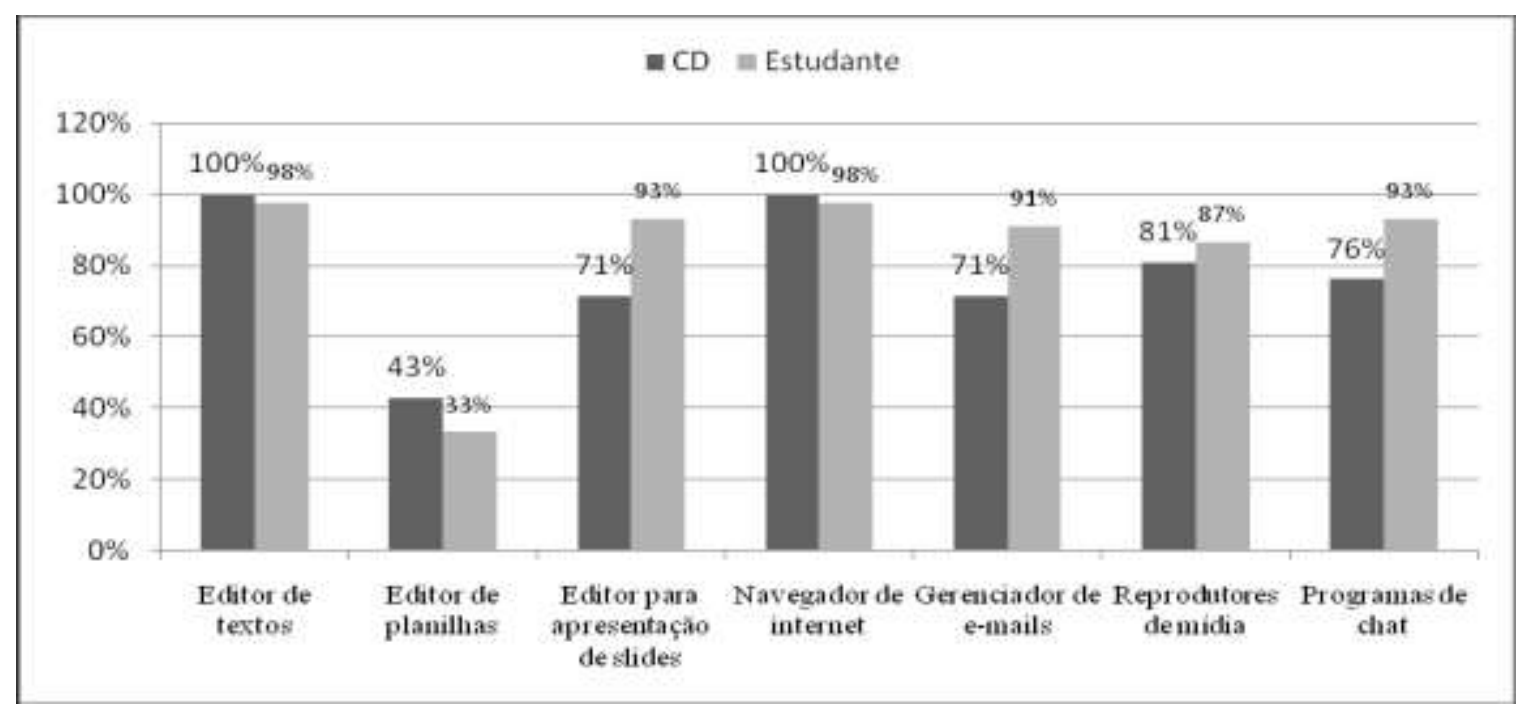

Fizeram acesso a página do Ministério da Saúde ou do DATASUS, pelo menos uma vez, $95,2 \%$ dos CD e $75.6 \%$ dos estudantes e realizaram acesso a página da $\mathrm{Bi}$ blioteca Virtual em Saúde (BVS) da BIREME $76.2 \%$ dos CD e $95.6 \%$ dos estudantes.

Conforme a proposta da questão aberta: "Durante a prática clínica diária pode surgir dúvidas com relação a diagnósticos e condutas clínicas a serem adotadas para determinados casos. Qual (ais) meio (os) você utiliza para resolver essas dúvidas durante o atendimento e após?" O exame qualitativo foi realizado por meio da técnica de Análise de Conteúdo. ${ }^{21}$ Para tal, fez-se uma leitura crítica das respostas e com esta, criou-se seis grupos categóricos. As catego- 
rias resultantes e a frequência de citações sentença, situadas no Quadro 1. pelos respondentes encontram-se ao final da

Quadro 1 - Meios que utilizam para resolver dúvidas surgidas durante o trabalho

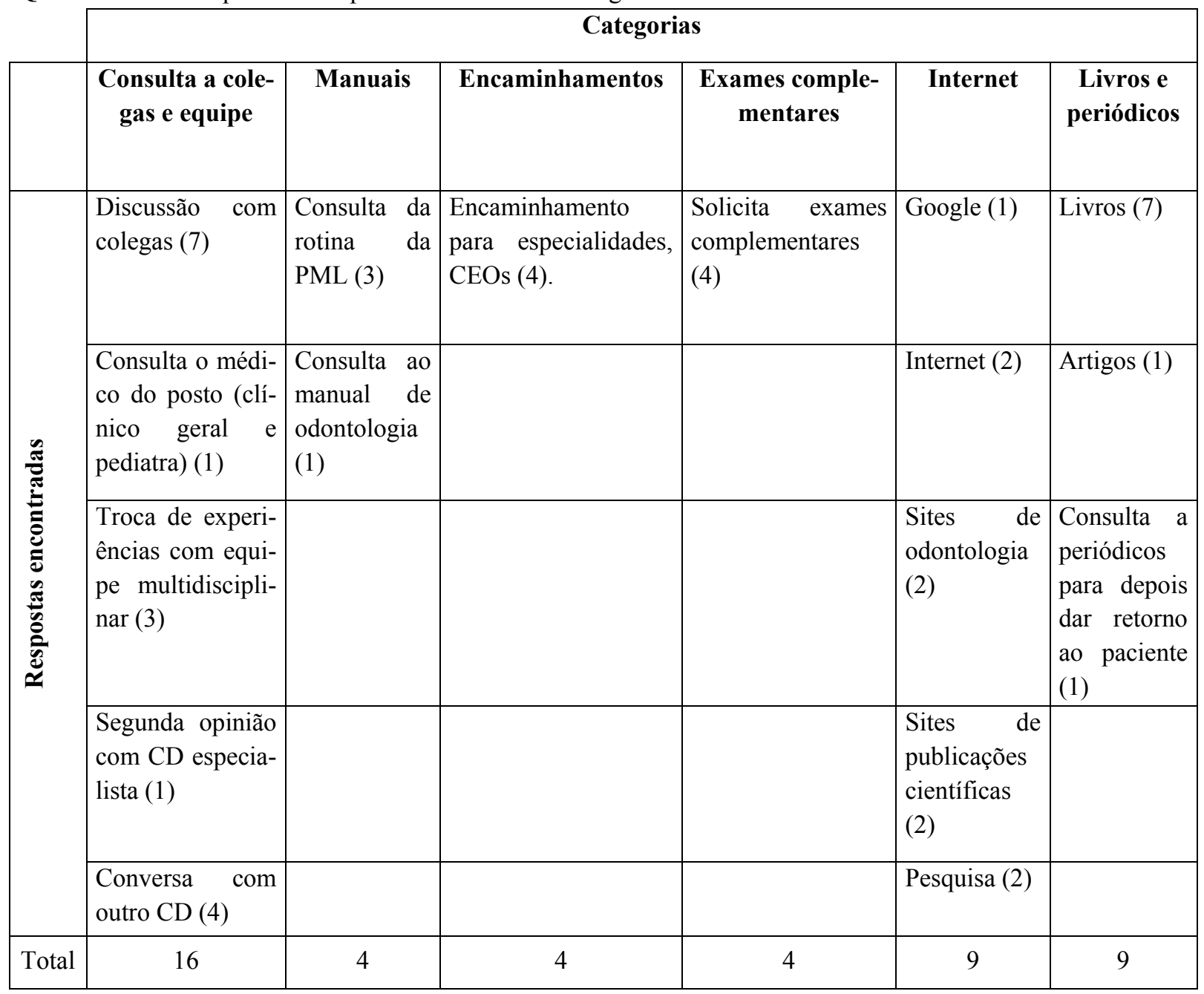

Analisando-se individualmente as resposta observou-se que, do total de respondentes, $12(57 \%)$ indivíduos não indicaram estudar ou obter informações mais precisas sobre o assunto em dúvida, buscam alternativas como a discussão com colegas, consulta a manuais ou realiza o encaminhamento para o nível secundário.

Todos os pesquisados em ambos os grupos relataram usar e-mail, com a seguinte frequência para o $\mathrm{CD}: 57,1 \%$ diariamente e $42,9 \%$ de 2 a 6 vezes por semana; para os estudantes: $62,2 \%$ diariamente, $28,9 \%$ de 2 a 6 vezes por semana, $8,8 \%$ frequências menores.

\section{DISCUSSÃO}

Observa-se no grupo pesquisado que mais de $75 \%$ dos $C D$ graduaram-se numa faixa entre 20 a 29 anos atrás, portanto com formação não direcionada para o atendimento em serviços públicos. Esse fato pode estar relacionado com a superespecialização característica do grupo, no qual $90 \%$ possui uma especialidade e $45 \%$ possui 2 especialidades, o que não é uma tendência nacional 
(25\% possuem 1 especialidade e apenas $3 \%$ possuem 2 especialidades). ${ }^{23}$

A superespecialização pode sugerir a dificuldade do profissional em resolver as dúvidas que vão surgindo no serviço, recorrendo-se a esses cursos como ferramenta de atualização de conhecimentos. Entende-se que há um esforço do profissional em compreender e se integrar ao ambiente de trabalho.

A formação continuada do profissional da saúde tem sido incentivada pelos órgãos gestores do SUS, como na Portaria 2.027 de 25 de agosto de 2011 que reserva, $8 \mathrm{~h}$ semanais para atividades de especialização na área e para educação permanente ${ }^{24,25}$ e por meio da Educação a Distância pela Universidade Aberta do SUS (UNA SUS). ${ }^{26}$

As DCN promovem a quebra do modelo de ensino Flexneriano, ${ }^{14}$ ao voltar nosso olhar para o estudante de graduação, como visto na Tabela 2 , preocupa o fato de que $77.7 \%$ pretendem fazer curso de Especialização ou Residência. Contudo, apenas um estudante citou a Saúde Coletiva e um Residência em Saúde da Família, mesmo entre os $29 \%$ que foram bolsistas do PETSaúde (destes, 38.5\% foram bolsistas por 2 anos) apenas dois se interessaram pela área.

Apesar dos avanços apresentados com a nova proposta de ensino, com os estágios curriculares no SUS, com o Programa Nacional de Reorientação da Formação Profissional em Saúde (Pró-Saúde) e com o PET-Saúde, os dados indicam que o novo currículo ainda não foi suficiente para mudar o perfil do estudante ao final da graduação, pois ainda continua voltado para o exercício privado da profissão. Especula-se que os estudantes pesquisados ainda não reconhecem a saúde pública como a importante área de trabalho que se afirmou nos últimos anos.
O fácil acesso ao conhecimento é um benefício da sociedade da informação, porém ressalta-se a importância de reconhecer e acessar conteúdos com qualidade científica.

Observa-se que nenhum dos $\mathrm{CD}$ e um estudante utiliza sites de Odontologia Baseada em Evidências (OBE), os quais possuem revisões sistemáticas e informações baseadas na melhor evidência científica disponível, inclusive com pesquisas clínicas atuais. Porém ao acessar os artigos de OBE e aqueles em periódicos de maior fator de impacto (revistas com alta qualidade científica), estão escritos em inglês.

Dos profissionais pesquisados $57.1 \%$ não apresenta proximidade com a leitura de textos em inglês e $53.4 \%$ dos estudantes. Conclui-se que para superar a barreira da informação de qualidade muitos leitores se deparam com a barreira da linguagem.

Apesar de muitos dos pesquisados marcarem utilizar Periódicos e livros para estudar, questiona-se o limitado alcance da informação que têm acessado, visto o desconhecimento da OBE, a falta de domínio da língua inglesa e o fato de $14.3 \%$ dos CD e $17.8 \%$ dos estudantes utilizar sites e blogs de $\mathrm{CD}$ (que geralmente não fornecem referências bibliográficas).

Ainda, após análise individual das respostas referentes ao Quadro 1, concluiuse que $3 \mathrm{CD}$ (14.3\% dos pesquisados) utilizam como métodos de estudo, exclusivamente: discussão de caso com colegas, grupo de estudo e palestras/congressos. $\mathrm{Ou}$ seja, não recorrem à literatura para resolver suas dúvidas (não marcaram periódicos, livros ou sites científicos). Aprofundando o obtido na questão fechada com o resultado da questão aberta direcionada ao $\mathrm{CD}$, obteve-se que $12(57 \%)$ indivíduos não relata- 
ram estudar para resolver a dúvida levantada durante o trabalho.

Ainda que se considere as limitações de um estudo baseado na auto percepção, a falta de cultura de busca de informações e conhecimento referenciada pelo grupo de cirurgiões-dentistas é um problema a ser superado. A autonomia é que possibilitará o enfrentamento das situações problema que surgirão no dia-a-dia do trabalho. Resolver as dúvidas que aparecem durante o serviço, por meio da leitura de artigos científicos, é uma premissa para o sucesso de estratégias de apoio ao profissional, tendo grande relação com o retorno em benefícios ao processo de trabalho e à população atendida.

Destaca-se a necessidade de uma contrapartida dos órgãos envolvidos em facilitar o acesso à informação de qualidade e fortalecer estratégias já adotadas, como no site da Biblioteca Virtual em Saúde no domínio da $\mathrm{APS}^{27}$ no qual se disponibiliza traduções de revisões sistemáticas de bases como a Cochrane, porém ainda há poucas traduções, principalmente referentes à Odontologia. É importante também, que os gestores e a equipe local, busquem alternativas para melhorar o processo de trabalho do $\mathrm{CD}$, de forma a contemplar tempo para atividades de estudo individual, conforme disposto na Portaria 2.027 de 25 de agosto de $2011,{ }^{25}$ o tempo para educação do profissional é elemento fundamental da qualidade da atenção no SUS e do compromisso ético em oferecer um tratamento de qualidade.

Tanto para o profissional quanto para o estudante, as estratégias pedagógicas devem favorecer a autonomia do indivíduo e sensibilizá-lo quanto à importância do seu papel proativo na resolução dos problemas de saúde e dos benefícios que a prática qualificada trará para aqueles atendidos. Para isso, as ações devem ser bem planejadas e se deve estabelecer ferramentas de acompanhamento, na forma de apoio às ações já desenvolvidas. Bem como, o professor também precisa renovar seus métodos de ensino, atuar como facilitador no processo ensino-aprendizagem e instigar no estudante o desejo de estudar e resolver suas dúvidas com base em evidências científicas de qualidade.

Considerando que $75 \%$ dos CD já acessaram os sites da BVS ou da Biblioteca Virtual em Saúde Pública, indica-se a possibilidade de utilizar dessas fontes como ferramenta de acesso a outras estratégias de apoio que poderão ser desenvolvidas, dado semelhante foi encontrado por Rendeiro et al. $(2012)^{28}$

Há anos a BVS tem disponibilizado conteúdo científico de qualidade e sua atuação por meio da BVS APS - Atenção Primária à Saúde promove uma importante inovação ao fornecer um conteúdo diferenciado para os profissionais que estão no serviço e necessitam de uma informação mais rápida e concisa, ${ }^{3}$ bem como, é em um dos domínios da BVS que as produções referentes ao Telessaúde estão disponíveis para consulta.

Expostas as vantagens do uso da educação à distância e das TIC, os grupos pesquisados mostraram-se bem integrados aos principais recursos de informática necessários para a utilização de uma ferramenta de suporte à distância, conforme o revelado na Figura 2. A quase totalidade de sujeitos que possuem e-mail o acessam com frequência, bem como o uso de ferramentas virtuais para estudar e se atualizar corroboram para a presença de um bom perfil infocultural nos grupos estudados, não se constituindo numa barreira de acesso.

$\mathrm{Na}$ pesquisa de Languardia et al. $(2010)^{8}$ os autores concluíram que não há necessidade de sofisticados conhecimentos 
de informática para trabalhar em um AVA, as ferramentas básicas são suficientes para os primeiros acessos e a correta e bem estruturada formulação do AVA é que permitirá o melhor aproveitamento dos recursos disponíveis. Contudo, a oferta de treinamento para o uso das TIC foi indicada como muito importante para a implementação do programa em estabelecimentos de saúde, conforme levantamento TIC Saúde 2013. ${ }^{29}$

Ao observar a Quadro 1, referente aos grupos categóricos resultantes da Análise de Conteúdo, levanta-se a questão dos encaminhamentos ao nível secundário de atenção (Centros de Especialidades Odontológicas-CEO).

Entre os objetivos do programa Telessaúde estão: tornar os atendimentos mais rápidos e melhorar a resolubilidade. A sobrecarga do nível secundário de atenção com encaminhamentos indevidos é um problema a ser resolvido. As características do grupo de CD pesquisados demonstram essa ocorrência, como visto no Quadro1.

Os resultados obtidos com o Telessaúde chamam atenção devido a grande capacidade de atuar nesse desafio. A experiência do programa no estado do Rio Grande do Sul concluiu que a disponibilização da Segunda Opinião Formativa evitou que $70 \%$ dos pacientes fossem referidos a outro serviço (serviço utilizado por médicos). Já no estado de Minas gerais a redução no encaminhamento foi de $78 \%$. Outro resultado importante arrolado pelo programa no estado de Minas Gerais foi a manutenção de profissionais da Equipe de Saúde na Família em regiões de difícil acesso, no qual $67 \%$ dos pesquisados considerou que a oferta de capacitação foi um fator de alta importância para a permanência no município longínquo. ${ }^{3}$ Com a perspectiva de expansão da Teleodontologia' o potencial de uso dessa estratégia na Odontologia necessita ser mais bem investigado.

Ressalta-se uma característica dos grupos pesquisados: a recorrência aos colegas como método auxiliar na resolução dos problemas levantados. É importante correlacionar essa característica com o desenho do Telessaúde, que disponibiliza apoio qualificado via internet aos profissionais. Ora, se esses recorrem aos colegas, a disponibilidade da Teleconsultoria associada à facilidade com que os pesquisados trabalham com novas tecnologias, apresenta-se como estratégia promissora.

\section{CONCLUSÕES}

Conclui-se que a maior barreira para programas de apoio ao profissional está na falta de uma cultura de busca de informação e de resolução de problemas embasados no conhecimento científico. Bem como, deve haver uma contrapartida dos programas em fornecer conteúdos de mais fácil acesso e com qualidade científica. Também se indica a premissa da graduação em Odontologia enfatizar a busca autônoma e qualificada da informação.

A intenção revelada pelos estudantes em obter uma especialidade, após a conclusão da graduação, mostra que despertar o interesse para o trabalho no SUS ainda é um desafio a ser superado. Apesar dos avanços conseguidos com as mudanças curriculares e com a criação de programas de inserção do estudante no ambiente de trabalho da saúde pública, essas ações ainda não foram suficientes para mudar o perfil do grupo de estudantes, que mostrou-se voltado para o exercício privado da profissão. 


\section{REFERÊNCIAS}

1. Brasil. Ministério da Saúde. Secretaria de Gestão do Trabalho e da Educação na Saúde. Departamento de Gestão da Educação em Saúde. Política Nacional de Educação Permanente em Saúde. Brasília: Ministério da Saúde, 2009. 64 p.

2. Luiz RR, Costa AJL, Nadavosky P. Epidemiologia e Bioestatística em Odontologia. São Paulo: Atheneu, 2008.

3. Haddad AE. Experiência Brasileira do Programa Nacional Telessaúde Brasil. Gold book [recurso eletrônico]: inovação tecnológica em educação e saúde. Rio de Janeiro: EdUERJ, 2012. p.1244.Disponível

em:http://www.telessaude.uerj.br/goldb ook/artigos/Acesso em: 01/11/2013.

4. Oliveira ESG; Rego, MCLC; Villardi, RM. Aprendizagem mediada por ferramentas de interação: análise do discurso de professores em um curso de formação continuada à distância. Educ. Soc., Campinas, vol. 28, n. 101, p. 1413-1434, set./dez. 2007.

5. Castells M. A galáxia da internet: reflexões sobre a internet, negócios e a sociedade. Rio de Janeiro: Jorge Zahar Ed., 2003. 238p. (P.7)

6. Lévy P. As Tecnologias da Inteligência. O Futuro do Pensamento na Era da Informática. Rio de Janeiro: Ed. 34, 2001.

7. França G. Os ambientes de aprendizagem na época da hipermídia e da Educação à Distância. Perspectivas em Ciência da Informação. V.14, n.1, p.5565, Jan/abr, 2009.

8. Languardia J, Casanova A, Machado R. A experiência de aprendizagem online em um curso de qualificação pro- fissional em saúde. Trab. Educ. Saúde, Rio de Janeiro, v.8, n.1, p.97-122, mar/jun 2010.

9. Brasil. Decreto $\mathrm{n}^{\mathrm{o}} 7.175$ de 12 de maio de 2010. Programa Nacional de Banda Larga (PNBL) - Brasil Conectado. Disponível em: http://www4.planalto.gov.br/brasilcone ctado/pnbl Acesso em: 20/01/2014

10. Almeida MEB.Educação à distância na internet: abordagens e contribuições dos ambientes digitais de aprendizagem.Educação e Pesquisa, São Paulo, v.29, n.2, p. 327-340, jul./dez. 2003.

11. Belloni ML. Educação a Distância. Editora Autores Associados. 4ed.

12. Brasil. Conselho Nacional de Educação. Câmara de Educação Superior. Resolução CNE/CES 3, de 19 de fevereiro de 2002. Institui Diretrizes Curriculares Nacionais do Curso de Graduação em Odontologia. Diário Oficial União, seção1:10, 04 mar 2002. Disponível em: http://portal.mec.gov.br/cne/arquivos/p df/CES032002.pdf Acesso em: 20/01/2014.

13. Freire, P. Pedagogia do Oprimido. Editora Paz e Terra, 1970.

14. Almeida Filho N. Reconhecer Flexner: inquérito sobre produção de mitos na educação médica no Brasil contemporâneo. Cad. Saúde Pública, Rio de Janeiro, 26(12):2234-2249, dez, 2010. P.2235

15. Perrenoud P. Construir as competências desde a escola. Porto Alegre: Artmed, 1999. p. 19-33.

16. Brasil. Ministério da Saúde. Telessaúde Brasil Redes. Disponível em: 
http://www.telessaudebrasil.org.br/ Acesso em: 01/11/2013.

17. Haddad AE, Brenelli SL, Passarella TM, Ribeiro TCV. Política Nacional de Educação na Saúde. Revista Baiana de Saúde Pública. v.32, supl.1, p.98-114, out. 2008.

18. Brasil. Conselho Nacional de Secretários de Saúde. Atenção Primária e Promoção da Saúde / Conselho Nacional de Secretários de Saúde. - Brasília: CONASS, 2007. $232 \mathrm{p}$.

19. Brasil. Ministério da Saúde. Portaria ${ }^{\circ}$ 2.546/GM/MS de 27 de outubro de 2011. Redefine e amplia o Programa Telessaúde Brasil, que passa a ser denominado Programa Nacional Telessaúde Brasil Redes (Telessaúde Brasil Redes). Brasília: Diário Oficial da União, Seção 1, Página 48, de 08 de Novembro de 2011.

20. Universidade Estadual de Londrina. Resolução CEPE n ${ }^{\circ} 45$ de 2005. Reformula o Projeto Pedagógico do Curso de Odontologia, a ser implantado a partir do ano letivo de 2005. Londrina, 2005. Disponível em: http://www.uel.br/prograd/pp/documen tos/odontologia.pdf Acesso em: 14/02/3014.

21. Telessaúde Brasil. Ministério da Saúde. Formulário Linha de Base. Site: $<<$ www.tele.medicina.ufg.br/file/LB_Odo nto.pdf >> Acesso em: 04/02/2013

22. Bardin L. Análise de conteúdo. Lisboa: Edições 70, 1977.

23. Morita MC, Haddad AE, Araújo ME. Perfil atual e tendências do Cirurgião Dentista brasileiro. Maringá: Dental Press International, 2010. 96p.
24. Brasil. Ministério da Saúde. Ministério da Educação. Portaria interministerial $\mathrm{n}^{\mathrm{o}} 2.117$ de 3 de novembro de 2005 . Institui no âmbito dos Ministérios da Saúde e da Educação, a Residência Multiprofissional em Saúde e dá outras providências. Brasília: Diário Oficial da União no 212, Seção 1, Página 112, de 4 de novembro de 2005.

25. Brasil. Ministério da Saúde. Portaria $\mathrm{N}^{\mathrm{o}}$ 2.027, DE 25 de agosto de 2011. Altera a Portaria $n^{\circ}$ 648/GM/MS, de 28 de março de 2006, na parte que dispõe sobre a carga horária dos profissionais médicos que compõem as Equipes de Saúde da Família (ESF) e na parte que dispõe sobre a suspensão do Piso de Atenção Básica (PAB Variável). Disponível em: http://bvsms.saude.gov.br/bvs/saudeleg is../gm/2011/prt2027_25_08_2011.htm 1 Acesso em: 15/11/2013.

26. Brasil. Portal da Saúde. Universidade Aberta do Sistema Único de Saúde. Disponível em: http://portal.saude.gov.br/portal/saude/ visualizar_texto.cfm?idtxt $=29886$

Acesso em: 01/11/2013.

27. Brasil. Biblioteca Virtual em Saúde. BVS APS: Atenção Primária à Saúde. Revisões Sistemáticas traduzidas. Disponível em: http://pearls.telessaudebrasil.org.br/ Acesso em: 26/01/2014.

28. Rendeiro MMP, Vieira C, Figueiredo DLTM. Infocultura: uma barreira de acesso à educação permanente? Gold book [recurso eletrônico]: inovação tecnológica em educação e saúde. Rio de Janeiro: EdUERJ, 2012. p.832-845Disponível 
em:http://www.telessaude.uerj.br/goldb ook/artigos/Acesso em:15/11/2013.

29. Centro de Estudos sobre as Tecnologias da Informação e da Comunicação. Pesquisa sobre o Uso das Tecnologias da Informação e da Comunicação no Brasil. Disponível em: http://cetic.br/saude/2013/ Acesso em: 15/02/2014.

\section{AGRADECIMENTOS}

CAPES- Bolsa de Mestrado concedida à Primeira autora no

Edital PróEnsino na Saúde, 2012-2014.

\section{ABSTRACT \\ Study tools used by dentistry students and Dentists to study and to resolve doubts emerged from the work}

The objective was to describe tools and means used by a group of 45 students and 21 dentists, working at the Public Health System and student's tutor, to study and solve questions raised during working. Furthermore, it aimed to identify barriers for virtual environments as support strategy. Semi-structured questionnaire was applied and items were analyzed using descriptive statistics and Content Analysis. The main studying tools for students were electronic journals $(89 \%)$, for the dentists was attending seminars and conferences (91\%). Both groups mastered basic computer tools, email and digital searching tools. Websites and blogs were used by $14.3 \%$ of dentists and $17.8 \%$ of students. None of dentist and only one student indicated using EvidenceBased Dentistry sites. Reading English papers is essential for the access to the scientific knowledge, it was a barrier to over $50 \%$ for both groups. The qualitative approach found that $57 \%$ of the dentists indicated they are not used to study to resolve questions emerged from the work, solved mainly with conversations between colleagues and referrals. It shows promise for the strategies such as teleconsulting. Although the desired alumni profile is the general practioner, the intention of specializing is reported by $78 \%$ students. They mastered the technological field, however the barrier related to the lack of a searching culture, may affect adherence to distance support programs. The research indicates the need to emphasize the constant searching for qualified information, for students and dentists, leading to the resolution and quality of care offered in the public health services.

Descriptors: Continuing Education. Education, Dental. Distance Education. EvidenceBased Dentistry, Telemedicine. 